\title{
NONMONOTONE IMPULSE EFFECTS IN SECOND-ORDER PERIODIC BOUNDARY VALUE PROBLEMS
}

\author{
IRENA RACHŮNKOVÁ AND MILAN TVRDÝ
}

Received 23 September 2002

We deal with the nonlinear impulsive periodic boundary value problem $u^{\prime \prime}=f\left(t, u, u^{\prime}\right)$, $u\left(t_{i}+\right)=\mathrm{J}_{i}\left(u\left(t_{i}\right)\right), u^{\prime}\left(t_{i}+\right)=\mathrm{M}_{i}\left(u^{\prime}\left(t_{i}\right)\right), i=1,2, \ldots, m, u(0)=u(T), u^{\prime}(0)=u^{\prime}(T)$. We establish the existence results which rely on the presence of a well-ordered pair $\left(\sigma_{1}, \sigma_{2}\right)$ of lower/upper functions $\left(\sigma_{1} \leq \sigma_{2}\right.$ on $\left.[0, T]\right)$ associated with the problem. In contrast to previous papers investigating such problems, the monotonicity of the impulse functions $\mathrm{J}_{i}$, $\mathrm{M}_{i}$ is not required here.

\section{Introduction}

In recent years, the theory of impulsive differential equations has become a well-respected branch of mathematics. This is because of its characteristic features which provide many interesting problems that cannot be solved by applying standard methods from the theory of ordinary differential equations. It can also give a natural description of many real models from applied sciences (see the examples mentioned in $[1,2]$ ).

In particular, starting with [7], periodic boundary value problems for nonlinear second-order impulsive differential equations of the form (2.1), (2.2), and (2.3) have received considerable attention; see, e.g., $[1,3,5,6,8,9,14]$, where the existence results in terms of lower and upper functions can also be found. However, all impose certain monotonicity requirements on the impulse functions. In contrast to these papers, we provide existence results using weaker conditions (2.10) and (2.11) instead of monotonicity.

Throughout the paper, we keep the following notation and conventions. For a real valued function $u$ defined a.e. on $[0, T]$, we put

$$
\|u\|_{\infty}=\sup _{t \in[0, T]} \operatorname{ess}|u(t)|, \quad\|u\|_{1}=\int_{0}^{T}|u(s)| d s .
$$

For a given interval $J \subset \mathbb{R}$, let $\mathbb{C}(J)$ denote the set of real-valued functions which are continuous on $J$. Furthermore, let $\mathbb{C}^{1}(J)$ be the set of functions having continuous first derivatives on $J$, and $\mathbb{L}(J)$ the set of functions which are Lebesgue integrable on $J$. 
Let $m \in \mathbb{N}$ and let

$$
0=t_{0}<t_{1}<t_{2}<\cdots<t_{m}<t_{m+1}=T
$$

be a division of the interval $[0, T]$. We denote

$$
\mathrm{D}=\left\{t_{1}, t_{2}, \ldots, t_{m}\right\}
$$

and define $\mathbb{C}_{D}^{1}[0, T]$ as the set of functions $u:[0, T] \mapsto \mathbb{R}$,

$$
u(t)=\left\{\begin{array}{cc}
u_{[0]}(t) & \text { if } t \in\left[0, t_{1}\right], \\
u_{[1]}(t) & \text { if } t \in\left(t_{1}, t_{2}\right], \\
\vdots & \vdots \\
u_{[m]}(t) & \text { if } t \in\left(t_{m}, T\right],
\end{array}\right.
$$

where $u_{[i]} \in \mathbb{C}^{1}\left[t_{i}, t_{i+1}\right]$ for $i=0,1, \ldots, m$. Moreover, $\mathbb{A C}_{D}^{1}[0, T]$ stands for the set of functions $u \in \mathbb{C}_{\mathrm{D}}^{1}[0, T]$ having first derivatives absolutely continuous on each subinterval $\left(t_{i}, t_{i+1}\right), i=0,1, \ldots, m$. For $u \in \mathbb{C}_{\mathrm{D}}^{1}[0, T]$ and $i=1,2, \ldots, m+1$, we write

$$
\begin{gathered}
u^{\prime}\left(t_{i}\right)=u^{\prime}\left(t_{i}-\right)=\lim _{t \rightarrow t_{i}-} u^{\prime}(t), \quad u^{\prime}(0)=u^{\prime}(0+)=\lim _{t \rightarrow 0+} u^{\prime}(t), \\
\|u\|_{\mathrm{D}}=\|u\|_{\infty}+\left\|u^{\prime}\right\|_{\infty} .
\end{gathered}
$$

Note that the set $\mathbb{C}_{\mathrm{D}}^{1}[0, T]$ becomes a Banach space when equipped with the norm $\|\cdot\|_{D}$ and with the usual algebraic operations.

We say that $f:[0, T] \times \mathbb{R}^{2} \mapsto \mathbb{R}$ satisfies the Carathéodory conditions on $[0, T] \times \mathbb{R}^{2}$ if

(i) for each $x \in \mathbb{R}$ and $y \in \mathbb{R}$, the function $f(\cdot, x, y)$ is measurable on $[0, T]$;

(ii) for a.e. $t \in[0, T]$, the function $f(t, \cdot, \cdot)$ is continuous on $\mathbb{R}^{2}$;

(iii) for each compact set $K \subset \mathbb{R}^{2}$, there is a function $m_{K}(t) \in \mathbb{L}[0, T]$ such that $|f(t, x, y)| \leq m_{K}(t)$ holds for a.e. $t \in[0, T]$ and all $(x, y) \in K$.

The set of functions satisfying the Carathéodory conditions on $[0, T] \times \mathbb{R}^{2}$ will be denoted by $\operatorname{Car}\left([0, T] \times \mathbb{R}^{2}\right)$.

Given a Banach space $\mathbb{X}$ and its subset $M$, let $\operatorname{cl}(M)$ and $\partial M$ denote the closure and the boundary of $M$, respectively.

Let $\Omega$ be an open bounded subset of $\mathbb{X}$. Assume that the operator $\mathrm{F}: \operatorname{cl}(\Omega) \mapsto \mathbb{X}$ is completely continuous and $\mathrm{F} u \neq u$ for all $u \in \partial \Omega$. Then $\operatorname{deg}(\mathrm{I}-\mathrm{F}, \Omega)$ denotes the LeraySchauder topological degree of $\mathrm{I}-\mathrm{F}$ with respect to $\Omega$, where I is the identity operator on $\mathbb{X}$. For a definition and properties of the degree, see, for example, [4] or [10].

\section{Formulation of the problem and main assumptions}

Here we study the existence of solutions to the following problem:

$$
\begin{gathered}
u^{\prime \prime}=f\left(t, u, u^{\prime}\right), \\
u\left(t_{i}+\right)=\mathrm{J}_{i}\left(u\left(t_{i}\right)\right), \quad u^{\prime}\left(t_{i}+\right)=\mathrm{M}_{i}\left(u^{\prime}\left(t_{i}\right)\right), \quad i=1,2, \ldots, m, \\
u(0)=u(T), \quad u^{\prime}(0)=u^{\prime}(T),
\end{gathered}
$$


where $u^{\prime}\left(t_{i}\right)$ are understood in the sense of (1.5), $f \in \operatorname{Car}\left([0, T] \times \mathbb{R}^{2}\right), \mathrm{J}_{i} \in \mathbb{C}(\mathbb{R})$, and $\mathrm{M}_{i} \in \mathbb{C}(\mathbb{R})$.

Definition 2.1. A solution of the problem (2.1), (2.2), and (2.3) is a function $u \in \mathbb{A} \mathbb{C}_{D}^{1}[0, T]$ which satisfies the impulsive conditions (2.2), the periodic conditions (2.3), and for a.e. $t \in[0, T]$ fulfils the equation $u^{\prime \prime}(t)=f\left(t, u(t), u^{\prime}(t)\right)$.

Definition 2.2. A function $\sigma_{1} \in \mathbb{A C}_{D}^{1}[0, T]$ is called a lower function of problem (2.1), (2.2), and (2.3) if

$$
\begin{gathered}
\sigma_{1}^{\prime \prime}(t) \geq f\left(t, \sigma_{1}(t), \sigma_{1}^{\prime}(t)\right) \quad \text { for a.e. } t \in[0, T], \\
\sigma_{1}\left(t_{i}+\right)=\mathrm{J}_{i}\left(\sigma_{1}\left(t_{i}\right)\right), \quad \sigma_{1}^{\prime}\left(t_{i}+\right) \geq \mathrm{M}_{i}\left(\sigma_{1}^{\prime}\left(t_{i}\right)\right), \quad i=1,2, \ldots, m, \\
\sigma_{1}(0)=\sigma_{1}(T), \quad \sigma_{1}^{\prime}(0) \geq \sigma_{1}^{\prime}(T) .
\end{gathered}
$$

Similarly, a function $\sigma_{2} \in \mathbb{A C}_{\mathrm{D}}^{1}[0, T]$ is an upper function of problem (2.1), (2.2), and (2.3) if

$$
\begin{gathered}
\sigma_{2}^{\prime \prime}(t) \leq f\left(t, \sigma_{2}(t), \sigma_{2}^{\prime}(t)\right) \quad \text { for a.e. } t \in[0, T], \\
\sigma_{2}\left(t_{i}+\right)=\mathrm{J}_{i}\left(\sigma_{2}\left(t_{i}\right)\right), \quad \sigma_{2}^{\prime}\left(t_{i}+\right) \leq \mathrm{M}_{i}\left(\sigma_{2}^{\prime}\left(t_{i}\right)\right), \quad i=1,2, \ldots, m, \\
\sigma_{2}(0)=\sigma_{2}(T), \quad \sigma_{2}^{\prime}(0) \leq \sigma_{2}^{\prime}(T) .
\end{gathered}
$$

Throughout the paper we assume

$$
\begin{gathered}
0=t_{0}<t_{1}<t_{2}<\cdots<t_{m}<t_{m+1}=T<\infty, \quad \mathrm{D}=\left\{t_{1}, t_{2}, \ldots, t_{m}\right\}, \\
f \in \operatorname{Car}\left([0, T] \times \mathbb{R}^{2}\right), \quad \mathrm{J}_{i} \in \mathbb{C}(\mathbb{R}), \quad \mathrm{M}_{i} \in \mathbb{C}(\mathbb{R}), \quad i=1,2, \ldots, m ;
\end{gathered}
$$

$\sigma_{1}$ and $\sigma_{2}$ are, respectively, lower and upper functions of (2.1), (2.2), and (2.3),

$$
\begin{gathered}
\sigma_{1} \leq \sigma_{2} \text { on }[0, T] ; \\
\sigma_{1}\left(t_{i}\right) \leq x \leq \sigma_{2}\left(t_{i}\right) \Longrightarrow \mathrm{J}_{i}\left(\sigma_{1}\left(t_{i}\right)\right) \leq \mathrm{J}_{i}(x) \leq \mathrm{J}_{i}\left(\sigma_{2}\left(t_{i}\right)\right), \quad i=1,2, \ldots, m ; \\
y \leq \sigma_{1}^{\prime}\left(t_{i}\right) \Longrightarrow \mathrm{M}_{i}(y) \leq \mathrm{M}_{i}\left(\sigma_{1}^{\prime}\left(t_{i}\right)\right), \\
y \geq \sigma_{2}^{\prime}\left(t_{i}\right) \Longrightarrow \mathrm{M}_{i}(y) \geq \mathrm{M}_{i}\left(\sigma_{2}^{\prime}\left(t_{i}\right)\right), \quad i=1,2, \ldots, m
\end{gathered}
$$

Remark 2.3. If $\mathrm{M}_{i}(0)=0$ for $i=1,2, \ldots, m$ and $r_{1} \in \mathbb{R}$ is such that $\mathrm{J}_{i}\left(r_{1}\right)=r_{1}$ for $i=$ $1,2, \ldots, m$ and

$$
f\left(t, r_{1}, 0\right) \leq 0 \quad \text { for a.e. } t \in[0, T]
$$

then $\sigma_{1}(t) \equiv r_{1}$ on $[0, T]$ is a lower function of problem (2.1), (2.2), and (2.3). Similarly, if $r_{2} \in \mathbb{R}$ is such that $\mathrm{J}_{i}\left(r_{2}\right)=r_{2}$ for all $i=1,2, \ldots, m$ and

$$
f\left(t, r_{2}, 0\right) \geq 0 \quad \text { for a.e. } t \in[0, T]
$$

then $\sigma_{2}(t) \equiv r_{2}$ is an upper function of problem (2.1), (2.2), and (2.3). 
580 Nonmonotone impulse effects in periodic problems

\section{A priori estimates}

At the beginning of this section, we introduce a class of auxiliary problems and prove uniform a priori estimates for their solutions.

Take $d \in \mathbb{R}, \tilde{f} \in \operatorname{Car}\left([0, T] \times \mathbb{R}^{2}\right), \widetilde{\mathrm{J}}_{i} \in \mathbb{C}(\mathbb{R})$, and $\widetilde{\mathrm{M}}_{i} \in \mathbb{C}(\mathbb{R}), i=1,2, \ldots, m$, such that

$$
\begin{aligned}
& \tilde{f}(t, x, y)<f\left(t, \sigma_{1}(t), \sigma_{1}^{\prime}(t)\right) \quad \text { for a.e. } t \in[0, T] \text {, all } x \in\left(-\infty, \sigma_{1}(t)\right), \\
& \text { and all } y \in \mathbb{R} \text { such that }\left|y-\sigma_{1}^{\prime}(t)\right| \leq \frac{\sigma_{1}(t)-x}{\sigma_{1}(t)-x+1} ; \\
& \tilde{f}(t, x, y)>f\left(t, \sigma_{2}(t), \sigma_{2}^{\prime}(t)\right) \quad \text { for a.e. } t \in[0, T], \text { all } x \in\left(\sigma_{2}(t), \infty\right), \\
& \text { and all } y \in \mathbb{R} \text { such that }\left|y-\sigma_{2}^{\prime}(t)\right| \leq \frac{x-\sigma_{2}(t)}{x-\sigma_{2}(t)+1} ; \\
& \widetilde{\mathrm{J}}_{i}(x)<\mathrm{J}_{i}\left(\sigma_{1}\left(t_{i}\right)\right) \quad \text { if } x<\sigma_{1}\left(t_{i}\right), \\
& \widetilde{\mathrm{J}}_{i}(x)=\mathrm{J}_{i}(x) \quad \text { if } x \in\left[\sigma_{1}\left(t_{i}\right), \sigma_{2}\left(t_{i}\right)\right], \quad(i=1,2, \ldots, m) ; \\
& \widetilde{\mathrm{J}}_{i}(x)>\mathrm{J}_{i}\left(\sigma_{2}\left(t_{i}\right)\right) \quad \text { if } x>\sigma_{2}\left(t_{i}\right), \\
& \widetilde{\mathrm{M}}_{i}(y) \leq \mathrm{M}_{i}\left(\sigma_{1}^{\prime}\left(t_{i}\right)\right) \quad \text { if } y \leq \sigma_{1}^{\prime}\left(t_{i}\right), \quad(i=1,2, \ldots, m) ; \\
& \widetilde{\mathrm{M}}_{i}(y) \geq \mathrm{M}_{i}\left(\sigma_{2}^{\prime}\left(t_{i}\right)\right) \quad \text { if } y \geq \sigma_{2}^{\prime}\left(t_{i}\right), \\
& \sigma_{1}(0) \leq d \leq \sigma_{2}(0),
\end{aligned}
$$

and consider an auxiliary Dirichlet problem

$$
\begin{array}{ll} 
& u^{\prime \prime}=\tilde{f}\left(t, u, u^{\prime}\right), \\
u\left(t_{i}+\right)=\widetilde{J}_{i}\left(u\left(t_{i}\right)\right), & u^{\prime}\left(t_{i}+\right)=\widetilde{M}_{i}\left(u^{\prime}\left(t_{i}\right)\right), \quad i=1,2, \ldots, m, \\
& u(0)=u(T)=d .
\end{array}
$$

Lemma 3.1. Let (2.8), (2.9), and (2.10) and (3.1), (3.2), (3.3), and (3.4) hold. Then every solution $u$ of (3.5), (3.6), and (3.7) satisfies

$$
\sigma_{1} \leq u \leq \sigma_{2} \quad \text { on }[0, T]
$$

Proof. Let $u$ be a solution of (3.5), (3.6), and (3.7). Put $v(t)=u(t)-\sigma_{2}(t)$ for $t \in[0, T]$. Then, by (3.4), we have

$$
v(0)=v(T) \leq 0
$$

So, it remains to prove that $v \leq 0$ on $(0, T)$.

Part (i). First, we show that $v$ does not have a positive local maximum at any point of $(0, T) \backslash \mathrm{D}$. Assume, on the contrary, that there is $\alpha \in(0, T) \backslash \mathrm{D}$ such that $v$ has a positive local maximum at $\alpha$, that is,

$$
v(\alpha)>0, \quad v^{\prime}(\alpha)=0 .
$$


This guarantees the existence of $\beta$ such that $[\alpha, \beta] \subset(0, T) \backslash D$ and

$$
v(t)>0, \quad\left|v^{\prime}(t)\right|<\frac{v(t)}{v(t)+1}<1
$$

for $t \in[\alpha, \beta]$. Using (2.5), (3.1), and (3.11), we get

$$
\begin{aligned}
v^{\prime \prime}(t) & =u^{\prime \prime}(t)-\sigma_{2}^{\prime \prime}(t)=\tilde{f}\left(t, u(t), u^{\prime}(t)\right)-\sigma_{2}^{\prime \prime}(t) \\
& >f\left(t, \sigma_{2}(t), \sigma_{2}^{\prime}(t)\right)-\sigma_{2}^{\prime \prime}(t) \geq 0
\end{aligned}
$$

for a.e. $t \in[\alpha, \beta]$. Hence,

$$
0<\int_{\alpha}^{t} v^{\prime \prime}(s) d s=v^{\prime}(t)
$$

for all $t \in(\alpha, \beta]$. This contradicts that $v$ has a local maximum at $\alpha$.

Part (ii). Now, assume that there is $t_{j} \in \mathrm{D}$ such that

$$
\max _{t \in\left(t_{j-1}, t_{j}\right]} v(t)=v\left(t_{j}\right)>0 .
$$

Then $v^{\prime}\left(t_{j}\right) \geq 0$. By (3.2) and (3.3), we get

$$
\widetilde{\mathrm{J}}_{j}\left(u\left(t_{j}\right)\right)>\mathrm{J}_{j}\left(\sigma_{2}\left(t_{j}\right)\right), \quad \widetilde{\mathrm{M}}_{j}\left(u^{\prime}\left(t_{j}\right)\right) \geq \mathrm{M}_{j}\left(\sigma_{2}^{\prime}\left(t_{j}\right)\right) .
$$

By (3.6) and (2.6), the relations

$$
v\left(t_{j}+\right)>0, \quad v^{\prime}\left(t_{j}+\right) \geq 0
$$

follow. If $v^{\prime}\left(t_{j}+\right)>0$, then there is $\beta \in\left(t_{j}, t_{j+1}\right)$ such that

$$
v^{\prime}(t)>0 \quad \text { on }\left(t_{j}, \beta\right] .
$$

If $v^{\prime}\left(t_{j}+\right)=0$, then we can find $\beta$ such that $\left(t_{j}, \beta\right] \subset(0, T) \backslash \mathrm{D}$ and (3.11) is satisfied on $\left(t_{j}, \beta\right]$. Consequently, (3.17) is valid in this case as well. As by Part (i) $v^{\prime}$ cannot change its sign on $\left(t_{j}, t_{j+1}\right)$, in both these cases we have

$$
v^{\prime}(t) \geq 0 \quad \text { on }\left(t_{j}, t_{j+1}\right) .
$$

Now, by (3.16),(3.17), and (3.18), we get

$$
\max _{t \in\left(t_{j}, t_{j+1}\right]} v(t)=v\left(t_{j+1}\right)>0 .
$$

Continuing inductively, we get $v(T)>0$ contrary to (3.9). 
582 Nonmonotone impulse effects in periodic problems

Part (iii). Finally, assume that

$$
\sup _{t \in\left(t_{j}, t_{j+1}\right]} v(t)=v\left(t_{j}+\right)>0
$$

for some $t_{j} \in \mathrm{D}$. In view of (3.2), this is possible only if

$$
\tilde{\mathrm{J}}_{j}\left(u\left(t_{j}\right)\right)>\mathrm{J}_{j}\left(\sigma_{2}\left(t_{j}\right)\right)
$$

If $u\left(t_{j}\right) \in\left[\sigma_{1}\left(t_{j}\right), \sigma_{2}\left(t_{j}\right)\right]$, then by (3.2) and (2.10), we have

$$
\widetilde{\mathrm{J}}_{j}\left(u\left(t_{j}\right)\right)=\mathrm{J}_{j}\left(u\left(t_{j}\right)\right) \leq \mathrm{J}_{j}\left(\sigma_{2}\left(t_{j}\right)\right),
$$

contrary to (3.21). If $u\left(t_{j}\right)<\sigma_{1}\left(t_{j}\right)$, then by (3.2), (2.9), and (2.10), we get

$$
\tilde{\mathrm{J}}_{j}\left(u\left(t_{j}\right)\right)<\mathrm{J}_{j}\left(\sigma_{1}\left(t_{j}\right)\right) \leq \mathrm{J}_{j}\left(\sigma_{2}\left(t_{j}\right)\right),
$$

again a contradiction to (3.21). Therefore, $u\left(t_{j}\right)>\sigma_{2}\left(t_{j}\right)$, that is, $v\left(t_{j}\right)>0$. Further, (3.20) gives $v^{\prime}\left(t_{j}+\right) \leq 0$. If $v^{\prime}\left(t_{j}+\right)=0$, then, as in Part (ii), we get (3.17), which contradicts (3.20). Therefore, $v^{\prime}\left(t_{j}+\right)<0$, which yields, with (3.3), that $v^{\prime}\left(t_{j}\right)<0$. Thus, in view of Part (i), we deduce that $v^{\prime} \leq 0$ on $\left(t_{j-1}, t_{j}\right)$, that is, $\sup _{t \in\left(t_{j-1}, t_{j}\right]} v(t)=v\left(t_{j-1}+\right)>0$. Continuing inductively, we get $v(0)>0$, contradicting (3.9).

To summarize, we have proved that $v \leq 0$ on $[0, T]$ which means that $u \leq \sigma_{2}$ on $[0, T]$. If we put $v=\sigma_{1}-u$ on $[0, T]$ and use the properties of $\sigma_{1}$ instead of $\sigma_{2}$, we can prove $\sigma_{1} \leq u$ on $[0, T]$ by an analogous argument.

In the proof of Theorem 4.1, we need a priori estimates for derivatives of solutions. To this aim we prove the following lemma.

Lemma 3.2. Assume that $r \in(0, \infty)$ and that

$$
\begin{gathered}
h \in \mathbb{L}[0, T] \quad \text { is nonnegative a.e. on }[0, T], \\
\omega \in \mathbb{C}([1, \infty)) \quad \text { is positive on }[1, \infty), \quad \int_{1}^{\infty} \frac{d s}{\omega(s)}=\infty .
\end{gathered}
$$

Then there exists $r^{*} \in(1, \infty)$ such that the estimate

$$
\left\|u^{\prime}\right\|_{\infty} \leq r^{*}
$$

holds for each function $u \in \mathbb{A C}_{D}^{1}[0, T]$ satisfying $\|u\|_{\infty} \leq r$ and

$$
\left|u^{\prime \prime}(t)\right| \leq \omega\left(\left|u^{\prime}(t)\right|\right)\left(\left|u^{\prime}(t)\right|+h(t)\right) \quad \text { for a.e. } t \in[0, T], \text { for }\left|u^{\prime}(t)\right|>1 \text {. }
$$

Proof. Let $u \in \mathbb{A C}_{D}^{1}[0, T]$ satisfy (3.27) and let $\|u\|_{\infty} \leq r$. The mean value theorem implies that there are $\xi_{i} \in\left(t_{i}, t_{i+1}\right)$ such that

$$
\left|u^{\prime}\left(\xi_{i}\right)\right|<\frac{2 r}{\Delta}+1, \quad i=1,2, \ldots, m,
$$


where

$$
\Delta=\min _{i=0,1, \ldots, m}\left(t_{i+1}-t_{i}\right)
$$

Put

$$
c_{0}=\frac{2 r}{\Delta}+1, \quad \rho=\left\|u^{\prime}\right\|_{\infty} .
$$

By replacing $u$ by $-u$ if necessary, we may assume that $\rho>c_{0}$ and

$$
\rho=\sup _{t \in\left(t_{j}, t_{j+1}\right]} u^{\prime}(t) \quad \text { for some } j \in\{0,1, \ldots, m\} .
$$

Thus we have

$$
\rho=u^{\prime}(\alpha) \quad \text { for some } \alpha \in\left(t_{j}, t_{j+1}\right]
$$

or

$$
\rho=u^{\prime}(\alpha+) \text { with } \quad \alpha=t_{j} .
$$

By (3.28), there is $\beta \in\left(t_{j}, t_{j+1}\right), \beta \neq \alpha$, such that $u^{\prime}(\beta)=c_{0}$ and $u^{\prime}(t) \geq c_{0}$ for all $t$ lying between $\alpha$ and $\beta$. Assume that (3.32) occurs. There are two cases to consider: $t_{j}<\beta<\alpha \leq$ $t_{j+1}$ or $t_{j}<\alpha<\beta<t_{j+1}$.

Case 1. Let $t_{j}<\beta<\alpha \leq t_{j+1}$. Since $u^{\prime}(t)>1$ on $[\beta, \alpha]$, (3.27) gives

$$
u^{\prime \prime}(t) \leq \omega\left(u^{\prime}(t)\right)\left(u^{\prime}(t)+h(t)\right) \quad \text { for a.e. } t \in[\beta, \alpha],
$$

and hence

$$
\int_{c_{0}}^{\rho} \frac{d s}{\omega(s)}=\int_{\beta}^{\alpha} \frac{u^{\prime \prime}(t)}{\omega\left(u^{\prime}(t)\right)} d t \leq \int_{\beta}^{\alpha} u^{\prime}(t) d t+\|h\|_{1} \leq 2 r+\|h\|_{1} .
$$

On the other hand, by (3.25), there is $r^{*}>c_{0}$ such that

$$
\int_{c_{0}}^{r^{*}} \frac{d s}{\omega(s)}>2 r+\|h\|_{1},
$$

which is possible only if $\rho<r^{*}$, that is, if (3.26) holds.

Case 2. Let $t_{j}<\alpha<\beta<t_{j+1}$. By (3.27), we get

$$
\begin{gathered}
-u^{\prime \prime}(t) \leq \omega\left(u^{\prime}(t)\right)\left(u^{\prime}(t)+h(t)\right) \quad \text { for a.e. } t \in[\alpha, \beta], \\
\int_{c_{0}}^{\rho} \frac{d s}{\omega(s)}=-\int_{\alpha}^{\beta} \frac{u^{\prime \prime}(t)}{\omega\left(u^{\prime}(t)\right)} d t \leq 2 r+\|h\|_{1},
\end{gathered}
$$

so the inequality (3.26) follows.

If (3.33) occurs, a similar argument to that in Case 2 applies and gives (3.26) as well. 
584 Nonmonotone impulse effects in periodic problems

Remark 3.3. Notice that the condition

$$
\int_{1}^{\infty} \frac{d s}{\omega(s)}=\infty
$$

in (3.25) can be weakened. In particular, the estimate (3.26) holds whenever $r^{*} \in(0, \infty)$ is such that

$$
\int_{c_{0}}^{r^{*}} \frac{d s}{\omega(s)}>2 r+\|h\|_{1} .
$$

\section{Main results}

The main existence result for problem (2.1), (2.2), and (2.3) is provided by the following theorem.

Theorem 4.1. Assume that (2.8), (2.9), (2.10), and (2.11) hold. Further, let

$$
|f(t, x, y)| \leq \omega(|y|)(|y|+h(t)) \quad \text { for a.e. } t \in[0, T] \text { and all } x \in\left[\sigma_{1}(t), \sigma_{2}(t)\right],|y|>1 \text {, }
$$

where $h$ and $\omega$ fulfil (3.24) and (3.25). Then the problem (2.1), (2.2), and (2.3) has a solution u satisfying (3.8).

Before proving this theorem, we prove the next key proposition where we restrict ourselves to the case that $f$ is bounded by a Lebesgue integrable function.

Proposition 4.2. Assume that (2.8), (2.9), (2.10), and (2.11) hold. Further, let $m \in \mathbb{L}[0, T]$ be such that

$$
|f(t, x, y)| \leq m(t) \quad \text { for a.e. } t \in[0, T] \text { and all }(x, y) \in\left[\sigma_{1}(t), \sigma_{2}(t)\right] \times \mathbb{R} \text {. }
$$

Then the problem (2.1), (2.2), and (2.3) has a solution u fulfilling (3.8).

Proof

Step 1. We construct a proper auxiliary problem.

Let $\Delta$ be given by (3.29). Put

$$
c=\|m\|_{1}+\frac{\left\|\sigma_{1}\right\|_{\infty}+\left\|\sigma_{2}\right\|_{\infty}}{\Delta}+\left\|\sigma_{1}^{\prime}\right\|_{\infty}+\left\|\sigma_{2}^{\prime}\right\|_{\infty},
$$

and for $t \in[0, T]$ and $(x, y) \in \mathbb{R}^{2}$, define

$$
\begin{gathered}
\alpha(t, x)= \begin{cases}\sigma_{1}(t) & \text { if } x<\sigma_{1}(t), \\
x & \text { if } \sigma_{1}(t) \leq x \leq \sigma_{2}(t), \\
\sigma_{2}(t) & \text { if } x>\sigma_{2}(t),\end{cases} \\
\beta(y)= \begin{cases}y & \text { if }|y| \leq c, \\
c \operatorname{sgn} y & \text { if }|y|>c .\end{cases}
\end{gathered}
$$


For a.e. $t \in[0, T]$ and all $(x, y) \in \mathbb{R}^{2}, \varepsilon \in[0,1]$, define functions

$$
\begin{gathered}
\omega_{k}(t, \varepsilon)=\sup _{y \in\left[\sigma_{k}^{\prime}(t)-\varepsilon, \sigma_{k}^{\prime}(t)+\varepsilon\right]}\left|f\left(t, \sigma_{k}(t), \sigma_{k}^{\prime}(t)\right)-f\left(t, \sigma_{k}(t), y\right)\right|, \quad k=1,2, \\
\widetilde{\mathrm{J}}_{i}(x)=x+\mathrm{J}_{i}\left(\alpha\left(t_{i}, x\right)\right)-\alpha\left(t_{i}, x\right), \quad i=1,2, \ldots, m \\
\widetilde{\mathrm{M}}_{i}(y)=y+\mathrm{M}_{i}(\beta(y))-\beta(y), \\
\tilde{f}(t, x, y)= \begin{cases}f\left(t, \sigma_{1}(t), y\right)-\omega_{1}\left(t, \frac{\sigma_{1}(t)-x}{\sigma_{1}(t)-x+1}\right)-\frac{\sigma_{1}(t)-x}{\sigma_{1}(t)-x+1} & \text { if } x<\sigma_{1}(t), \\
f(t, x, y) & \text { if } \sigma_{1}(t) \leq x \leq \sigma_{2}(t), \\
f\left(t, \sigma_{2}(t), y\right)+\omega_{2}\left(t, \frac{x-\sigma_{2}(t)}{x-\sigma_{2}(t)+1}\right)+\frac{x-\sigma_{2}(t)}{x-\sigma_{2}(t)+1} & \text { if } x>\sigma_{2}(t) .\end{cases}
\end{gathered}
$$

We see that $\omega_{k} \in \operatorname{Car}([0, T] \times[0,1])$ are nonnegative and nondecreasing in the second variable and $\omega_{k}(0)=0$ for $k=1,2$. Consequently, $\tilde{f} \in \operatorname{Car}\left([0, T] \times \mathbb{R}^{2}\right)$. Furthermore, $\tilde{\mathrm{J}}_{i}$, $\widetilde{\mathrm{M}}_{i} \in \mathbb{C}(\mathbb{R}), i=1,2, \ldots, m$. The auxiliary problem is $(3.5),(3.6)$, and

$$
u(0)=u(T)=\alpha\left(0, u(0)+u^{\prime}(0)-u^{\prime}(T)\right) .
$$

Step 2. We prove that problem (3.5), (3.6), (4.9) is solvable.

Let

$$
\begin{gathered}
G(t, s)= \begin{cases}\frac{t(s-T)}{T} & \text { if } 0 \leq t \leq s \leq T, \\
\frac{s(t-T)}{T} & \text { if } 0 \leq s<t \leq T,\end{cases} \\
G_{1}(t, s)= \begin{cases}-\frac{t}{T} & \text { if } 0 \leq t \leq s \leq T, \\
\frac{T-t}{T} & \text { if } 0 \leq s<t \leq T .\end{cases}
\end{gathered}
$$

Define an operator $\widetilde{\mathrm{F}}: \mathbb{C}_{\mathrm{D}}^{1}[0, T] \mapsto \mathbb{C}_{\mathrm{D}}^{1}[0, T]$ by

$$
\begin{aligned}
(\widetilde{\mathrm{F}} u)(t)= & \alpha\left(0, u(0)+u^{\prime}(0)-u^{\prime}(T)\right)+\int_{0}^{T} G(t, s) \tilde{f}\left(s, u(s), u^{\prime}(s)\right) d s \\
& +\sum_{i=1}^{m} G_{1}\left(t, t_{i}\right)\left(\widetilde{\mathrm{J}}_{i}\left(u\left(t_{i}\right)\right)-u\left(t_{i}\right)\right)+\sum_{i=1}^{m} G\left(t, t_{i}\right)\left(\widetilde{\mathrm{M}}_{i}\left(u^{\prime}\left(t_{i}\right)\right)-u^{\prime}\left(t_{i}\right)\right) .
\end{aligned}
$$

As in [13, Lemma 3.1], we get that $\widetilde{\mathrm{F}}$ is completely continuous and $u$ is a solution of (3.5), (3.6), (4.9) if and only if $u$ is a fixed point of $\tilde{\mathrm{F}}$.

Denote by $I$ the identity operator on $\mathbb{C}_{D}^{1}[0, T]$ and consider the parameter system of operator equations

$$
(\mathrm{I}-\lambda \widetilde{\mathrm{F}}) u=0, \quad \lambda \in[0,1] .
$$

For $R \in(0, \infty)$, define $\mathrm{B}(R)=\left\{u \in \mathbb{C}_{\mathrm{D}}^{1}[0, T]:\|u\|_{\mathrm{D}}<R\right\}$. By (4.2), (4.4), (4.5), (4.6), (4.7), (4.8), and (4.11), we can find $R_{0} \in(0, \infty)$ such that $u \in \mathrm{B}\left(R_{0}\right)$ for each $\lambda \in[0,1]$ 
586 Nonmonotone impulse effects in periodic problems

and each solution $u$ of (4.12). So, for each $R \geq R_{0}$, the operator $\mathrm{I}-\lambda \widetilde{\mathrm{F}}$ is a homotopy on $\operatorname{cl}(\mathrm{B}(R)) \times[0,1]$ and its Leray-Schauder degree $\operatorname{deg}(\mathrm{I}-\lambda \widetilde{\mathrm{F}}, \mathrm{B}(R))$ has the same value for each $\lambda \in[0,1]$. Since $\operatorname{deg}(\mathrm{I}, \mathrm{B}(R))=1$, we conclude that

$$
\operatorname{deg}(\mathrm{I}-\widetilde{\mathrm{F}}, \mathrm{B}(R))=1 \quad \text { for } R \in\left[R_{0}, \infty\right)
$$

By (4.13), there is at least one fixed point of $\widetilde{\mathrm{F}}$ in $\mathrm{B}(R)$. Hence there exists a solution of the auxiliary problem (3.5), (3.6), (4.9).

Step 3. We find estimates for solutions of the auxiliary problem.

Let $u$ be a solution of (3.5), (3.6), (4.9). We derive an estimate for $\|u\|_{\infty}$. By (4.7), (4.8), and (2.11), we obtain that $\tilde{f}, \widetilde{\mathrm{J}}_{i}, \widetilde{\mathrm{M}}_{i}, i=1,2, \ldots, m$, satisfy (3.1), (3.2), and (3.3). Moreover, in view of (4.4), we have

$$
\sigma_{1}(0) \leq \alpha\left(0, u(0)+u^{\prime}(0)-u^{\prime}(T)\right) \leq \sigma_{2}(0)
$$

Thus $u$ satisfies (3.8) by Lemma 3.1.

We find an estimate for $\left\|u^{\prime}\right\|_{\infty}$. By the mean value theorem and (3.8), there are $\xi_{i} \in$ $\left(t_{i}, t_{i+1}\right)$ such that

$$
\left|u^{\prime}\left(\xi_{i}\right)\right| \leq \frac{\left\|\sigma_{1}\right\|_{\infty}+\left\|\sigma_{2}\right\|_{\infty}}{\Delta}, \quad i=1,2, \ldots, m
$$

Moreover, by (3.8) and (4.8), $u$ satisfies (2.1) for a.e. $t \in[0, T]$. Therefore, integrating (2.1) and using (4.2), (4.3), and (4.15), we obtain

$$
\left\|u^{\prime}\right\|_{\infty} \leq\left|u^{\prime}\left(\xi_{i}\right)\right|+\|m\|_{1}<c
$$

Hence, by (4.7) and (4.9), we see that $u$ fulfils (2.2) and $u(0)=u(T)$ (i.e., the first condition from (2.3) is satisfied).

Step 4. We verify that $u$ fulfils the second condition in (2.3).

We must prove that $u^{\prime}(0)=u^{\prime}(T)$. By (4.9), this is equivalent to

$$
\sigma_{1}(0) \leq u(0)+u^{\prime}(0)-u^{\prime}(T) \leq \sigma_{2}(0)
$$

Suppose, on the contrary, that (4.17) is not satisfied. Let, for example,

$$
u(0)+u^{\prime}(0)-u^{\prime}(T)>\sigma_{2}(0)
$$

Then, by (4.4), we have $\alpha\left(0, u(0)+u^{\prime}(0)-u^{\prime}(T)\right)=\sigma_{2}(0)$. Together with (2.7) and (4.9), this yields

$$
u(0)=u(T)=\sigma_{2}(0)=\sigma_{2}(T)
$$


Inserting (4.19) into (4.18), we get

$$
u^{\prime}(0)>u^{\prime}(T)
$$

On the other hand, (4.19) together with (3.8) and (4.20) implies that

$$
\sigma_{2}^{\prime}(0) \geq u^{\prime}(0)>u^{\prime}(T) \geq \sigma_{2}^{\prime}(T)
$$

a contradiction to $(2.7)$.

If we assume that $u(0)+u^{\prime}(0)-u^{\prime}(T)<\sigma_{1}(0)$, we can argue similarly and again derive a contradiction to (2.7).

So, we have proved that (4.17) is valid, which means that $u^{\prime}(0)=u^{\prime}(T)$. Consequently, $u$ is a solution of (2.1), (2.2), and (2.3) satisfying (3.8).

Proof of Theorem 4.1. Put

$$
c=r^{*}+\left\|\sigma_{1}^{\prime}\right\|_{\infty}+\left\|\sigma_{2}^{\prime}\right\|_{\infty}
$$

where $r^{*} \in(0, \infty)$ is given by Lemma 3.2 for $r=\left\|\sigma_{1}\right\|_{\infty}+\left\|\sigma_{2}\right\|_{\infty}$. For a.e. $t \in[0, T]$ and all $(x, y) \in \mathbb{R}^{2}$, define a function

$$
g(t, x, y)= \begin{cases}f(t, x, y) & \text { if }|y| \leq c \\ \left(2-\frac{|y|}{c}\right) f(t, x, y) & \text { if } c<|y|<2 c \\ 0 & \text { if }|y| \geq 2 c\end{cases}
$$

Then $\sigma_{1}$ and $\sigma_{2}$ are, respectively, lower and upper functions of the auxiliary problem (2.2), (2.3), and

$$
u^{\prime \prime}=g\left(t, u, u^{\prime}\right)
$$

There exists a function $m^{*} \in \mathbb{L}[0, T]$ such that

$$
|f(t, x, y)| \leq m^{*}(t)
$$

for a.e. $t \in[0, T]$ and all $(x, y) \in\left[\sigma_{1}(t), \sigma_{2}(t)\right] \times[-2 c, 2 c]$. Hence

$$
|g(t, x, y)| \leq m^{*}(t) \quad \text { for a.e. } t \in[0, T] \text {, all }(x, y) \in\left[\sigma_{1}(t), \sigma_{2}(t)\right] \times \mathbb{R} \text {. }
$$

Since $g \in \operatorname{Car}\left([0, T] \times \mathbb{R}^{2}\right)$, we can apply Proposition 4.2 to problem (4.24), (2.2), (2.3) and get that this problem has a solution $u$ fulfilling (3.8). Hence $\|u\|_{\infty} \leq r$. Moreover, by (4.1), $u$ satisfies (3.27). Therefore, by Lemma 3.2, $\left\|u^{\prime}\right\|_{\infty} \leq r^{*} \leq c$. This implies that $u$ is a solution of (2.1), (2.2), and (2.3).

The next simple existence criterion, which follows from Theorem 4.1 and Remark 2.3, extends both [5, Theorem 4] and [13, Corollary 3.4]. 
588 Nonmonotone impulse effects in periodic problems

Corollary 4.3. Let (2.8) hold. Furthermore, assume that

(i) $\mathrm{M}_{i}(0)=0$ and $y \mathrm{M}_{i}(y) \geq 0$ for $y \in \mathbb{R}$ and $i=1,2, \ldots, m$;

(ii) there are $r_{1}, r_{2} \in \mathbb{R}$ such that $r_{1}<r_{2}, f\left(t, r_{1}, 0\right) \leq 0 \leq f\left(t, r_{2}, 0\right)$ for a.e. $t \in[0, T]$, $\mathrm{J}_{i}\left(r_{1}\right)=r_{1}, \mathrm{~J}_{i}(x) \in\left[r_{1}, r_{2}\right]$ if $x \in\left[r_{1}, r_{2}\right], \mathrm{J}_{i}\left(r_{2}\right)=r_{2}, i=1,2, \ldots, m$;

(iii) there are $h$ and $\omega$ satisfying (3.24) and (3.25) with $\sigma_{1}(t) \equiv r_{1}$ and $\sigma_{2}(t) \equiv r_{2}$ and such that (4.1) holds.

Then the problem (2.1), (2.2), and (2.3) has a solution $u$ fulfilling $r_{1} \leq u \leq r_{2}$ on $[0, T]$.

Remark 4.4. Let $\sigma_{1}<\sigma_{2}$ on $[0, T]$ and $\sigma_{1}\left(t_{i}+\right)<\sigma_{2}\left(t_{i}+\right)$ for $i=1,2, \ldots, m$. Having $G$ and $G_{1}$ from the proof of Proposition 4.2 , we define an operator $\mathrm{F}: \mathbb{C}_{\mathrm{D}}^{1}[0, T] \mapsto \mathbb{C}_{\mathrm{D}}^{1}[0, T]$ by

$$
\begin{aligned}
(\mathrm{F} u)(t)= & u(0)+u^{\prime}(0)-u^{\prime}(T)+\int_{0}^{T} G(t, s) f\left(s, u(s), u^{\prime}(s)\right) d s \\
& +\sum_{i=1}^{m} G_{1}\left(t, t_{i}\right)\left(\mathrm{J}_{i}\left(u\left(t_{i}\right)\right)-u\left(t_{i}\right)\right)+\sum_{i=1}^{m} G\left(t, t_{i}\right)\left(\mathrm{M}_{i}\left(u^{\prime}\left(t_{i}\right)\right)-u^{\prime}\left(t_{i}\right)\right) .
\end{aligned}
$$

Let $r^{*}$ be given by Lemma 3.2 for $r=\left\|\sigma_{1}\right\|_{\infty}+\left\|\sigma_{2}\right\|_{\infty}$. Define a set

$$
\begin{array}{r}
\Omega=\left\{u \in \mathbb{C}_{\mathrm{D}}^{1}[0, T]:\left\|u^{\prime}\right\|_{\infty}<r^{*}, \sigma_{1}(t)<u(t)<\sigma_{2}(t) \text { for } t \in[0, T],\right. \\
\left.\sigma_{1}\left(t_{i}+\right)<u\left(t_{i}+\right)<\sigma_{2}\left(t_{i}+\right) \text { for } i=1,2, \ldots, m\right\} .
\end{array}
$$

As in [13, Lemma 3.1], we get that $\mathrm{F}$ is completely continuous and $u$ is a solution of (2.1), (2.2), and (2.3) if and only if $u$ is a fixed point of F. The proofs of Theorem 4.1 and Proposition 4.2 yield the following result about the Leray-Schauder degree of the operator I $-\mathrm{F}$ with respect to $\Omega$.

Corollary 4.5. Let $\sigma_{1}<\sigma_{2}$ on $[0, T]$ and $\sigma_{1}\left(t_{i}+\right)<\sigma_{2}\left(t_{i}+\right)$ for $i=1,2, \ldots, m$, and let the assumptions of Theorem 4.1 be satisfied. Further, assume that $\mathrm{F}$ and $\Omega$ are defined by (4.27) and (4.28), respectively. If $\mathrm{F} u \neq u$ for each $u \in \partial \Omega$, then

$$
\operatorname{deg}(\mathrm{I}-\mathrm{F}, \Omega)=1
$$

Proof. Consider $c$ and $g$ from the proof of Theorem 4.1 and define $\widetilde{\mathrm{J}}_{i}, \widetilde{\mathrm{M}}_{i}, i=1,2, \ldots, m$, and $\tilde{f}$ by (4.7) and (4.8), where we put $g$ instead of $f$. Define $\widetilde{F}$ by (4.11) and put $\Omega_{1}=$ $\left\{u \in \Omega: \sigma_{1}(0)<u(0)+u^{\prime}(0)-u^{\prime}(T)<\sigma_{2}(0)\right\}$. Suppose that $\mathrm{F} u \neq u$ for each $u \in \partial \Omega$. We have

$$
\mathrm{F}=\widetilde{\mathrm{F}} \text { on } \mathrm{cl}\left(\Omega_{1}\right)
$$

and

$$
(\mathrm{F} u=u, u \in \Omega) \Longrightarrow u \in \Omega_{1} .
$$


By the proof of Proposition 4.2, we have that each fixed point $u$ of $\tilde{\mathrm{F}}$ satisfies (3.8) and consequently $\|u\|_{\infty} \leq r$. Hence, by (4.1), (4.8), and (4.23),

$$
\left|u^{\prime \prime}(t)\right|=\left|g\left(t, u(t), u^{\prime}(t)\right)\right| \leq \omega\left(\left|u^{\prime}(t)\right|\right)\left(\left|u^{\prime}(t)\right|+h(t)\right)
$$

for a.e. $t \in[0, T]$ and for $\left|u^{\prime}(t)\right|>1$. Therefore Lemma 3.2 implies that $\left\|u^{\prime}\right\|_{\infty} \leq r^{*}$. So, by (2.3), $u \in \operatorname{cl}\left(\Omega_{1}\right)$. Now, choose $R$ in (4.13) such that $\mathrm{B}(R) \supset \Omega$. Then, by the excision property of the degree, we get

$$
\operatorname{deg}(I-F, \Omega)=\operatorname{deg}\left(I-F, \Omega_{1}\right)=\operatorname{deg}\left(I-\widetilde{F}, \Omega_{1}\right)=\operatorname{deg}(I-\widetilde{F}, B(R))=1,
$$

wherefrom, taking into account (4.30), we obtain (4.29).

Remark 4.6. Following the ideas from $[12,13]$, the evaluation of $\operatorname{deg}(\mathrm{I}-\mathrm{F}, \Omega)$ enables us to prove the existence of solutions to problem (2.1), (2.2), and (2.3) also for nonordered lower/upper functions. This will be included in our next paper [11].

\section{Acknowledgment}

The first author was supported by Grant 201/01/1451 of the Grant Agency of the Czech Republic and by the Council of Czech Government J14/98:153100011. The second author was supported by Grant 201/01/1199 of the Grant Agency of the Czech Republic.

\section{References}

[1] D. Bănov and P. Simeonov, Impulsive Differential Equations: Periodic Solutions and Applications, Pitman Monographs and Surveys in Pure and Applied Mathematics, vol. 66, Longman Scientific \& Technical, Harlow, 1993.

[2] A. Cabada and E. Liz, Boundary value problems for higher order ordinary differential equations with impulses, Nonlinear Anal. 32 (1998), no. 6, 775-786.

[3] A. Cabada, J. J. Nieto, D. Franco, and S. I. Trofimchuk, A generalization of the monotone method for second order periodic boundary value problem with impulses at fixed points, Dynam. Contin. Discrete Impuls. Systems 7 (2000), no. 1, 145-158.

[4] J. Cronin, Fixed Points and Topological Degree in Nonlinear Analysis, Mathematical Surveys, no. 11, American Mathematical Society, Rhode Island, 1964.

[5] Y. Dong, Periodic solutions for second order impulsive differential systems, Nonlinear Anal. 27 (1996), no. 7, 811-820.

[6] L. H. Erbe and X. Liu, Existence results for boundary value problems of second order impulsive differential equations, J. Math. Anal. Appl. 149 (1990), no. 1, 56-69.

[7] S. Hu and V. Lakshmikantham, Periodic boundary value problems for second order impulsive differential systems, Nonlinear Anal. 13 (1989), no. 1, 75-85.

[8] E. Liz and J. J. Nieto, Periodic solutions of discontinuous impulsive differential systems, J. Math. Anal. Appl. 161 (1991), no. 2, 388-394.

[9] - The monotone iterative technique for periodic boundary value problems of second order impulsive differential equations, Comment. Math. Univ. Carolin. 34 (1993), no. 3, 405-411.

[10] J. Mawhin, Topological degree and boundary value problems for nonlinear differential equations, Topological Methods for Ordinary Differential Equations (M. Furi and P. Zecca, eds.), Lecture Notes in Math., vol. 1537, Springer-Verlag, Berlin, 1993, pp. 74-142.

[11] I. Rachůnková and M. Tvrdý, Non-ordered lower and upper functions in second order impulsive periodic problems, submitted to Dyn. Contin. Discrete Impuls. Syst. Ser. A Math. Anal. 
590 Nonmonotone impulse effects in periodic problems

[12] Nonlinear systems of differential inequalities and solvability of certain boundary value problems, J. Inequal. Appl. 6 (2001), no. 2, 199-226.

[13] Impulsive periodic boundary value problem and topological degree, Funct. Differ. Equ. 9 (2002), no. 3-4, 471-498.

[14] Z. Zhang, Existence of solutions for second order impulsive differential equations, Appl. Math. J. Chinese Univ. Ser. B 12 (1997), no. 3, 307-320.

Irena Rachůnková: Department of Mathematics, Palacký University, Tomkova 40, 77200 Olomouc, Czech Republic

E-mail address: rachunko@risc.upol.cz

Milan Tvrdý: Mathematical Institute, Academy of Sciences of the Czech Republic, Žitná 25, 11567 Praha 1, Czech Republic

E-mail address: tvrdy@math.cas.cz 


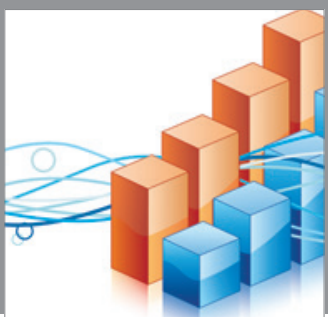

Advances in

Operations Research

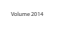

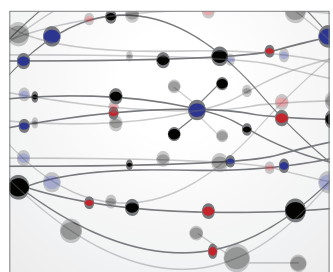

\section{The Scientific} World Journal
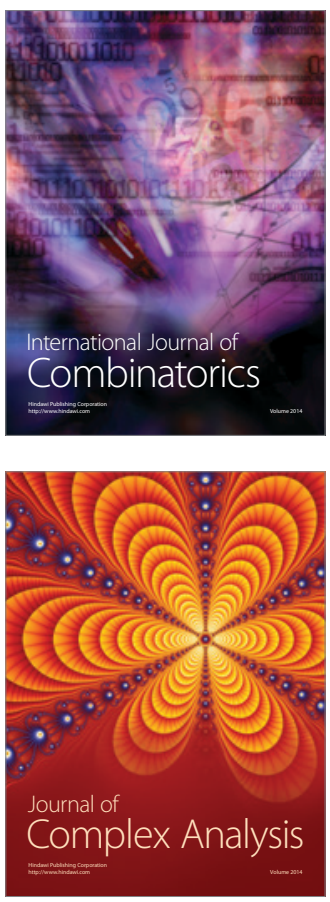

International Journal of

Mathematics and

Mathematical

Sciences
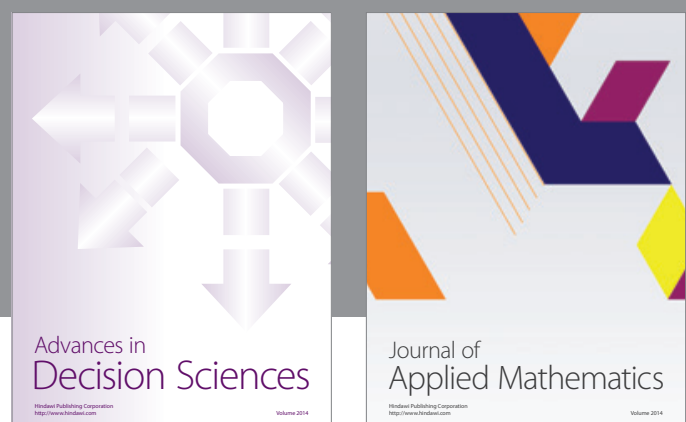

Journal of

Applied Mathematics
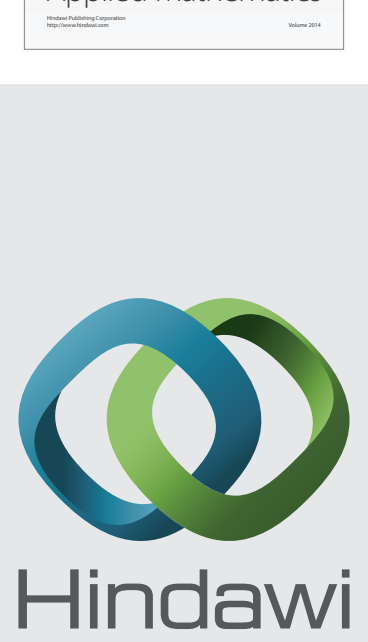

Submit your manuscripts at http://www.hindawi.com
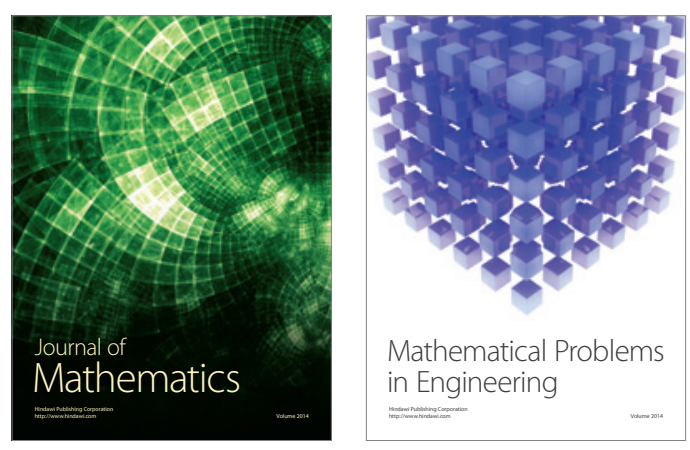

Mathematical Problems in Engineering
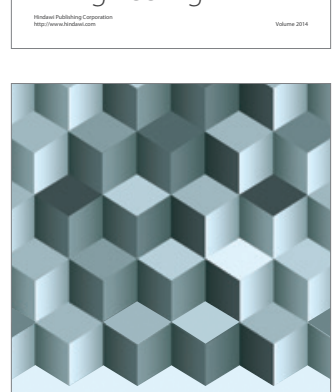

Journal of

Function Spaces
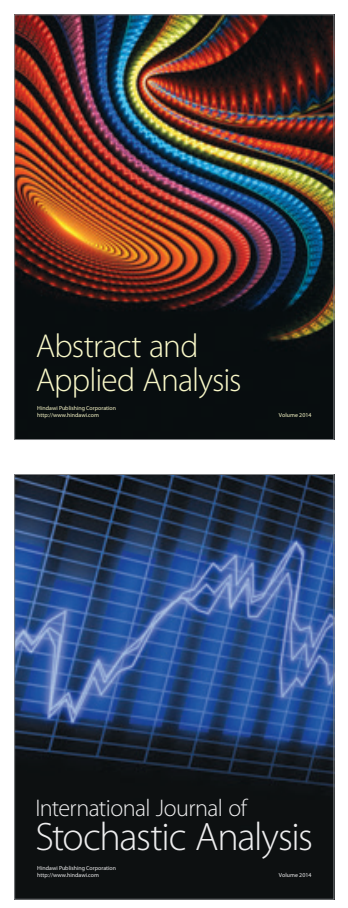

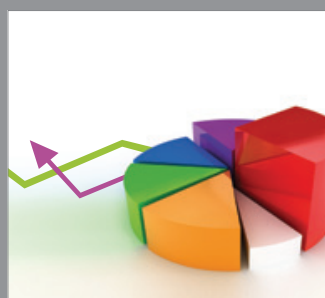

ournal of

Probability and Statistics

Promensencen
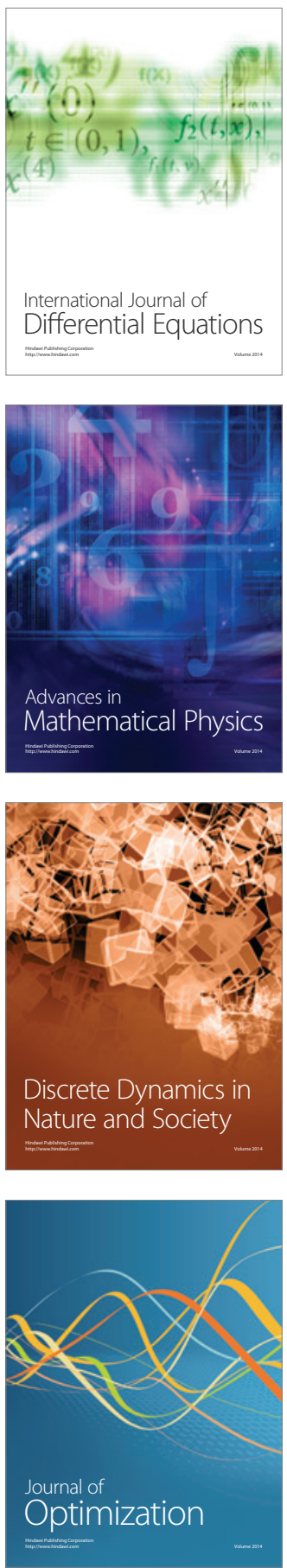